\title{
ON GROUPS WITH CAYLEY GRAPH ISOMORPHIC TO A CUBE
}

\author{
COLIN HAGEMEYER AND RICHARD SCOTT ${ }^{\dagger}$
}

\begin{abstract}
We say that a group $G$ is a cube group if it is generated by a set $S$ of involutions such that the corresponding Cayley graph Cay $(G, S)$ is isomorphic to a cube. Equivalently, $G$ is a cube group if it acts on a cube such that the action is simply-transitive on the vertices and the edge stabilizers are all nontrivial. The action on the cube extends to an orthogonal linear action, which we call the geometric representation. We prove a combinatorial decomposition for cube groups into products of 2-element subgroup, and show that the geometric representation is always reducible.
\end{abstract}

\section{Introduction}

Let $G$ be a group and let $S \subseteq G$ be a subset consisting of involutions. We say that the pair $(G, S)$ is a cube group (of rank $n$ ) if the corresponding Cayley graph Cay $(G, S)$ is isomorphic to the 1 -skeleton of the $n$-cube. Groups acting on CAT $(0)$ cube complexes such that the action is simply-transitive on vertices and has nontrivial edge stabilizers were studied in [1] as a natural generalization of right-angled Coxeter groups. Cube groups are precisely the finite groups in this class.

The purpose of this note is to prove two theorems about cube groups. The first is a product decomposition that implies the existence of a type of "boolean" normal form.

Theorem. Let $(G, S)$ be a cube group of rank $n$. For each $s \in S$, let $\langle s\rangle$ denote the subgroup generated by $s$. Then there exists an ordering $s_{1}, \ldots, s_{n}$ on the set $S$ such that

$$
G=\left\langle s_{1}\right\rangle\left\langle s_{2}\right\rangle \cdots\left\langle s_{n}\right\rangle .
$$

In particular, for any $g \in G$, there exist a unique choice of $m_{i} \in\{0,1\}, i=1, \ldots, n$ such that

$$
g=s_{1}^{m_{1}} s_{2}^{m_{2}} \cdots s_{n}^{m_{n}} .
$$

The action of a cube group $G$ on $\operatorname{Cay}(G, S)$ extends canonically to an isometric linear action on the full $n$-cube $[-1,1]^{n}$. We call this linear representation the geometric representation of $G$. The second theorem is a decomposition theorem for this representation.

Theorem. If $(G, S)$ is a cube group of rank $\geq 2$, then the geometric representation is reducible.

Both of these theorems are consequences of the following general fact about certain actions of $p$-groups, the proof of which is reminiscent of one of the standard combinatorial proofs of Sylow's theorem.

Both authors were supported by a Provost Office Grant from Santa Clara University.

$\dagger$ Corresponding author. 
Theorem. Let $X$ be a set with more than one element, and let $G$ be a p-group acting on $X$. If $G$ has a generating set such that every element fixes some element in $X$, then $X$ has at least two orbits.

\section{Decorated graphs and group presentations}

In this section, we described the relations among the generators of a cube group in terms of a certain graph with involutions. Let $S$ be a finite set, and let $\operatorname{Aut}(S)$ denote its permutation group. For each $s \in S$, let $j_{s} \in \operatorname{Aut}(S)$ be an involution satisfying $j_{s}(s)=s$. We can represent this data pictorially as follows. Let $K_{S}$ denote the complete graph with vertex set $S$. At each vertex $s$, draw an arc between all pairs of edges $\{s, u\}$ and $\{s, v\}$ whenever $j_{s}(u)=v$. We shall refer to such a choice of involutions $\Gamma=\left\{j_{s} \mid s \in S\right\}$ (and the resulting picture) as a decorated graph.

Example 2.1. For the decorated graph on $S=\{a, b, c, d, e\}$ shown in Figure 1, the involutions $j_{a}, j_{b}, j_{c}$, and $j_{d}$ are the transpositions $(b d),(a c),(b d)$, and $(a c)$, respectively. The involution $j_{e}$ is the product $(a c)(b d)$.

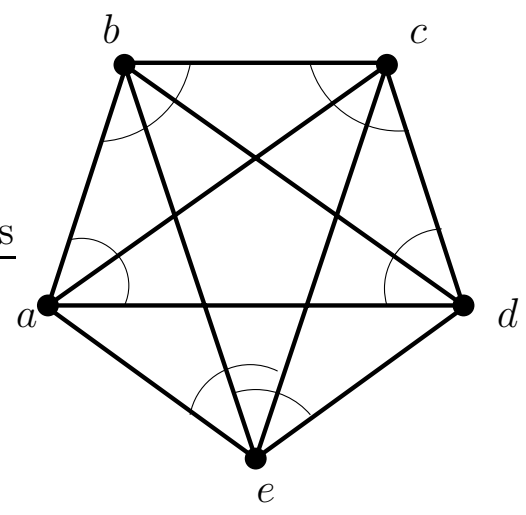

FiguRE 1.

Given a decorated graph $\Gamma$ on $S$ and any two distinct elements $s_{1}, s_{2} \in S$, one can define a sequence in $S$ inductively by $s_{n+1}=j_{s_{n}}\left(s_{n-1}\right)$. Such a sequence will be called a trajectory. A trajectory $s_{1}, s_{2}, \ldots$ is 4 -periodic if $s_{n}=s_{n+4}$ for all $n \geq 1$. We shall say the decorated graph has no holonomy along a trajectory $s_{1}, s_{2}, \ldots$ if $j_{s_{4}} \circ j_{s_{3}} \circ j_{s_{2}} \circ j_{s_{1}}$ is the identity permutation in $\operatorname{Aut}(S)$.

Definition 2.1. A decorated graph on $S$ is admissible if every trajectory is 4-periodic and has no holonomy along it.

The 4-periodicity condition simply means that the edges of a decorated graph (with their connecting arcs) can be partitioned into subsets of the form

- a 4-cycle with arcs joining consecutive edges,

- an angle (two edges meeting at a vertex joined by an arc), or

- a single edge (with no connecting arcs touching it). 
For example, the decorated graph in Figure 1 satisfies 4-periodicity since the edges can be partitioned as a single 4-cycle ( $\square a b c d)$, two angles ( $\angle a e c, \angle b e d)$, and two single edges $(a c$ and $b d)$. The no-holonomy condition is more difficult to verify but also holds for this decorated graph (for example, along a trajectory $a, b, c, d, a, b, c, d, \ldots$ corresponding to the 4-cycle, we have $\left.j_{d} \circ j_{c} \circ j_{b} \circ j_{a}=(a c)(b d)(a c)(b d)=\mathrm{Id}\right)$.

Given a decorated graph $\Gamma$ on $S$, we can define a group $W(\Gamma)$ by the presentation

$W(\Gamma)=\langle s \in S| s^{2}=1$ for all $s \in S$ and $s_{1} s_{2} s_{3} s_{4}=1$ for all trajectories $\left.s_{1}, s_{2}, \ldots\right\rangle$.

The following is a special case of the main result (Theorem 3.2) in [1].

Theorem 2.1. $(G, S)$ is a cube group if and only if there exists an admissible decorated graph $\Gamma$ on $S$ and an isomorphism $W(\Gamma) \rightarrow G$ that restricts to the identity on $S$.

We refer the reader to [1] for the full proof of this theorem, but indicate here how one obtains the decorated graph $\Gamma$ from the pair $(G, S)$.

Suppose $(G, S)$ is a cube group. Recall that the Cayley graph $\operatorname{Cay}(G, S)$ has vertex set $G$, and two vertices $g, g^{\prime}$ are joined by a directed edge if $g^{\prime}=g s$ for some $s \in S$. Each directed edge $(g, g s)$ is labeled by the element $s$, but since $s^{-1}=s$, we can unambiguously label the undirected edge $\{g, g s\}$ by the element $s$ as well. We shall therefore regard $\operatorname{Cay}(G, S)$ as an undirected graph on $G$ with edges labeled by elements of $S$. Recall that a presentation for $G$ can be obtained from the Cayley graph by taking products of labels around all cycles in the graph.

Since the Cayley graph for $G$ is isomorphic to a cube, cycles are generated by cycles of length 4, and any such 4-cycle can be translated by the (left) $G$-action (preserving labels) so that it starts at the identity vertex 1 . For a presentation, it therefore suffices to consider the 4-cycles touching the vertex 1 (and the trivial 2-cycles obtained by repeating an edge). At the vertex 1 , there are precisely $n$ incident edges, labeled by the elements of $S$. Any two elements $s_{1}, s_{2} \in S$, determine two such edges and hence span a unique 4-cycle (2-dimensional subface of the cube). Reading the labels around this 4-cycle, we obtain a relation $s_{1} s_{2} s_{3} s_{4}=1$.

To get the corresponding decorated graph $\Gamma$, we continue the relation to obtain a 4periodic sequence $s_{1}, s_{2}, s_{3}, s_{4}, s_{1}, \ldots$. These are the trajectories of our decorated graph $\Gamma$. To obtain the involutions $j_{s}, s \in S$, we simply consider all trajectories of the form $u, s, v, \ldots$ and define $j_{s}(u)=v$. One then needs to check that the resulting $j_{s}$ 's are well-defined involutions, and that there is no holonomy along trajectories.

Example 2.2. Let $G$ be the dihedral group of order 8, represented as the permutation group $G=\{\mathrm{Id},(13),(24),(13)(24),(12)(34),(14)(23),(1234),(1432)\}$. Letting $S=$ $\{a, b, c\}$ where $a=(13), b=(12)(34)$, and $c=(14)(23)$, we obtain the 3 -cube as the Cayley graph (Figure 2, left-hand side), hence $(G, S)$ is a cube group of rank 3 . Looking at pairs of edges incident to the vertex Id, we get the trajectories $a, b, a, c, \ldots$, $b, a, c, a, \ldots, a, c, a, b, \ldots, c, a, b, a, \ldots, b, c, b, c, \ldots$, and $c, b, c, b, \ldots$ The coresponding decorated graph on $S$ is therefore $\Gamma=\left\{j_{a}, j_{b}, j_{c}\right\}$ (Figure 2, right-hand side) where $j_{a}=(b c)$ and $j_{b}=j_{c}=\mathrm{Id}$, and we have a presentation for $G$ of the form

$$
W(\Gamma)=\left\langle a, b, c \mid a^{2}=b^{2}=c^{2}=1, a b a c=1, b c b c=1\right\rangle .
$$




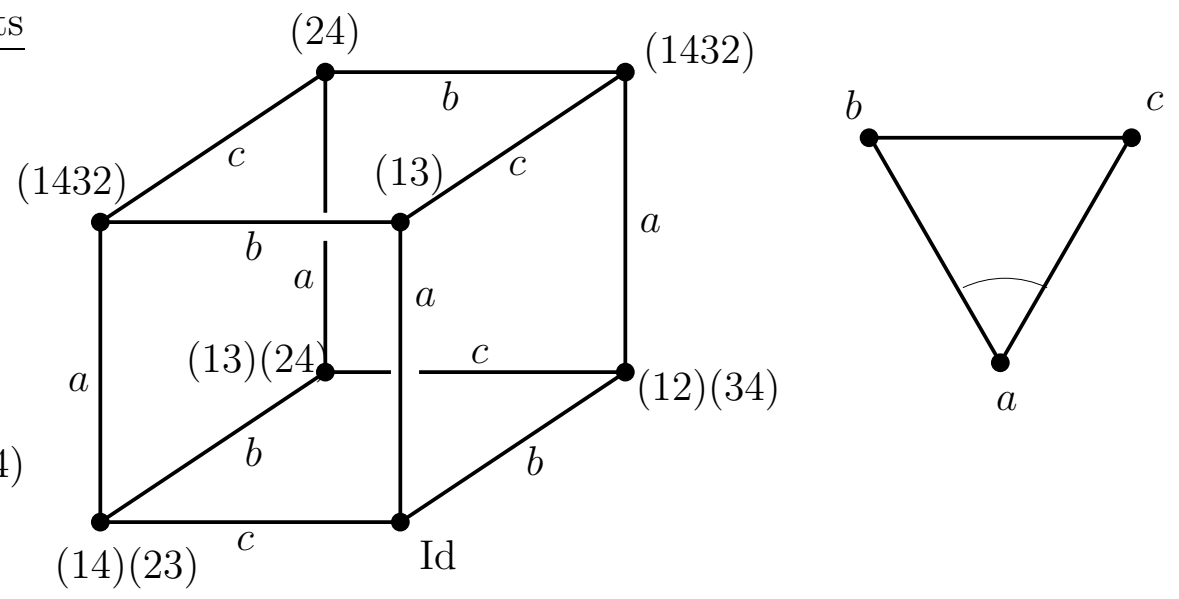

FIGURE 2.

\section{Product decompositions of cube groups}

Given a cube group $(G, S)$ any subset $T \subseteq S$ generates a subgroup of $G$ which we denote by $G_{T}$. We will call this subgroup a standard subgroup if $\left(G_{T}, T\right)$ is also a cube group.

Example 3.1. Let $(G, S)$ be the dihedral group in Example 2.2. The subgroup generated by $T=\{b, c\}$ is a standard subgroup since $\left(G_{T}, T\right)$ has Cayley graph isomorphic to a square. On the other hand, if $T=\{a, b\}$, then $\left(G_{T}, T\right)$ is not a standard subgroup. In this case $G_{T}$ is the entire group $(c=a b a)$ and $T$ has only two elements, hence the Cayley graph Cay $\left(G_{T}, T\right)$ will be an 8-cycle, which is not isomorphic to a cube.

In this section we describe how to decompose a cube group into products of standard subgroups. We use the fact that there is a natural action of the group $G$ on its generating set $S$.

Proposition 3.1. Let $(G, S)$ be a cube group with corresponding decorated graph $\Gamma=$ $\left\{j_{s} \mid s \in S\right\}$. Then the map $S \rightarrow \operatorname{Aut}(S)$ given by $s \mapsto j_{s}$ extends uniquely to a homomorphism $j: G \rightarrow \operatorname{Aut}(S)$.

Proof. One needs only check that the $j_{s}$ 's satisfy equations corresponding to the relations in the presentation $W(\Gamma)$. This is precisely the requirement that the $j_{s}$ 's be involutions and that $\Gamma$ have no holonomy along trajectories.

We shall call $j: S \rightarrow \operatorname{Aut}(S)$ the permutation representation for $(G, S)$. It defines an action of $G$ on the set $S$ by $g \cdot s=j_{g}(s)$ for all $g \in G$ and $s \in S$. Invariant subsets (i.e., unions of orbits) of this action give rise to product decompositions of $(G, S)$ with respect to standard subgroups.

Proposition 3.2. Let $(G, S)$ be a cube group and let $T \subseteq S$ be a $G$-invariant subset of $S$. Then $\left(G_{T}, T\right)$ is a standard subgroup and, moreover, we have a product decomposition

$$
G=G_{T} G_{S-T}
$$


(meaning any element $g \in G$ can be written uniquely in the form $g=g_{1} g_{2}$ where $g_{1} \in G_{T}$ and $\left.g_{2} \in G_{S-T}\right)$.

Proof. For any subset $T \subseteq S$, let $X_{T}$ denote the $|T|$-dimensional subcube of Cay $(G, S)$ containing the edges incident to 1 that are labeled by $t \in T$. To show that $G_{T}$ is a standard subgroup, it suffices to show that all of the labels on the edges of $X_{T}$ are in the subset $T$, since then $X_{T}$ will coincide with Cay $\left(G_{T}, T\right)$. For this, it is enough to show that the labels around any 4 -cycle in $X_{T}$ are always in $T$. Since $T$ is $G$-invariant, any trajectory starting $t_{1}, t_{2}, j_{t_{2}}\left(t_{1}\right), \ldots$ with $t_{1}, t_{2} \in T$ will have all terms in $T$, hence if a 4-cycle in Cay $\left(G_{T}, T\right)$ has 2 consecutive edges in $T$, it will have all edges in $T$. It follows that all 4-cycles in $X_{T}$ incident to the vertex 1 have edges labeled by elements of $T$, and then by induction on the distance to 1 that all 4-cycles in $X_{T}$ are labeled by elements of $T$.

Since $S-T$ will also be $G$-invariant, we have 2 standard subgroups $G_{T}$ and $G_{S-T}$. The subcubes $X_{T}$ and $X_{S-T}$ will intersect only in the vertex 1, hence the two subgroups $G_{T}$ and $G_{S-T}$ have trivial intersection. To prove the product decomposition, it is enough to show that any product st with $s \in S-T$ and $t \in T$ can be rewritten at $s t=t^{\prime} s^{\prime}$ with $t^{\prime} \in T$ and $s^{\prime} \in S-T$. Consider the trajectory $s, t, s^{\prime}, t^{\prime}, \ldots$ starting with $s$ and $t$. Then $s^{\prime}=j_{t}(s)$ and $t^{\prime}=t_{s^{\prime}}(t)$. Since $S-T$ is $G$-invariant and $s \in S-T$, we know $s^{\prime} \in S-T$, and since $T$ is $G$-invariant and $t \in T$, we know $t^{\prime} \in T$. On the other hand, the relation coming from this trajectory is $s t s^{\prime} t^{\prime}=1$, or (since generators are all involutions) $s t=t^{\prime} s^{\prime}$.

It turns out that for any cube group of rank at least 2, there is always a proper nontrivial invariant subset. This is the key technical result of this paper and uses the same counting argument found in one of the standard proofs of the first Sylow theorem (see [2]).

Theorem 3.1. Let $G$ be a p-group acting on a set $X$ with $|X| \geq 2$. Assume further that there is a generating subset $S \subseteq G$ such that for all $s \in S$ there exists an $x \in X$ such that $s \cdot x=x$. Then $X$ has at least two $G$-orbits.

Proof. Assume, on the contrary that the action is transitive on $X$. Since any subgroup of $G$ is also a $p$-group, the order of any orbit must also be a power of $p$, hence $|X|=p^{n}$ for some $n$. Let $\mathcal{U}$ denote the set of all subsets of $X$ of size $p^{n-1}$. Then

$$
|\mathcal{U}|=\left(\begin{array}{c}
p^{n} \\
p^{n-1}
\end{array}\right)=p \prod_{j=1}^{p^{n-1}-1} \frac{p^{n}-j}{p^{n-1}-j}=p \prod_{j=1}^{p^{n-1}-1} \frac{p^{n-\nu_{p}(j)}-j / p^{\nu_{p}(j)}}{p^{n-1-\nu_{p}(j)}-j / p^{\nu_{p}(j)}}
$$

where $\nu_{p}(j)$ denotes the $p$-adic valuation of $j$. Since none of the terms in the product on the right have any factors of $p$, we can write $|\mathcal{U}|=p m$ for some integer $m$ relatively prime to $p$.

Now consider the induced action of $G$ on $\mathcal{U}$. Since all orbits of this action must have order a power of $p$, and since the sum of these orders must be $p m$, there must be at least one orbit of size exactly $p$. Let $\mathcal{Y}$ be such an orbit. Then $\mathcal{Y}$ consists of $p$ subsets of $X$ of size exactly $p^{n-1}$, and since the action of $G$ on $X$ is transitive, these subsets form 
a partition of $X$. Given $x \in X$, we let $[x] \in \mathcal{Y}$ denote its equivalence class. Now, given $s \in S$, let $\langle s\rangle$ denote the subgroup it generates, and let $\mathcal{Y}^{\langle s\rangle}$ denote the elements of $\mathcal{Y}$ fixed by $\langle s\rangle$. Since there exists an $x \in X$ such that $s \cdot x=x$, we know that $s \cdot[x]=[x]$, hence $\mathcal{Y}^{\langle s\rangle}$ is not empty. On the other hand, since $\langle s\rangle$ is a $p$-group, we have

$$
\left|\mathcal{Y}^{\langle s\rangle}\right| \equiv|\mathcal{Y}| \bmod p \equiv p \bmod p \text {. }
$$

It follows that $\mathcal{Y}^{\langle s\rangle}=\mathcal{Y}$, so $s$ fixes $\mathcal{Y}$. Since this holds for all $s$ in the generating set $S$, the action of $G$ on $\mathcal{Y}$ must be trivial. But this contradicts transitivity of the action of $G$ on $X$

To apply this theorem (in the case $p=2$ ) to our setting, we suppose $(G, S)$ is a cube group with $|S| \geq 2$ and take $X=S$. The action of $G$ on $S$ has the property that $s \cdot s=j_{s}(s)=s$, hence every generator fixes some element. By Theorem 3.1 it follwos that the action has at least two orbits. Combining this with the previous proposition, we obtain our first theorem from the introduction.

Corollary 3.1. Let $(G, S)$ be a cube group of rank $n$. For each $s \in S$, let $\langle s\rangle$ denote the subgroup generated by $s$. Then there exists an ordering $s_{1}, \ldots, s_{n}$ on the set $S$ such that

$$
G=\left\langle s_{1}\right\rangle\left\langle s_{2}\right\rangle \cdots\left\langle s_{n}\right\rangle
$$

Proof. The proof is by induction on the rank of $S$. If $S=\{s\}$, then $G=\langle s\rangle$. In general, assume the decomposition holds for all cube groups of rank $<n$, and let $(G, S)$ be a cube group of rank $n$. By Theorem 3.1, there exists a proper, nontrivial $G$-invariant subset $T \subseteq S$. The groups $\left(G_{T}, T\right)$ and $\left(G_{S-T}, S-T\right)$ are cube groups of rank $<n$, say $k$ and $n-k$, respectively. Hence by induction, there exists an ordering $s_{1}, \ldots, s_{k}$ of the elements in $T$, and an ordering $s_{k+1}, \ldots, s_{n}$ of the elements in $S-T$, such that

$$
G_{T}=\left\langle s_{1}\right\rangle\left\langle s_{2}\right\rangle \cdots\left\langle s_{k}\right\rangle \text { and } G_{S-T}=\left\langle s_{k+1}\right\rangle\left\langle s_{k+2}\right\rangle \cdots\left\langle s_{n}\right\rangle .
$$

The result then follows from Proposition 3.2

Example 3.2. If $(G, S)$ is the dihedral group of Example 2.2, then $S$ breaks up into $G$-orbits $\{a\}$ and $T=\{b, c\}$, and $T$ breaks up into $G_{T}$-orbits $\{b\}$, and $\{c\}$. Hence,

$$
G=\langle a\rangle\langle b\rangle\langle c\rangle
$$

and we can take $a, b, c$ as our ordering on $S$. In other words, the elements of $G$ can be listed as $G=\{1, a, b, c, a b, a c, b c, a b c\}$. On the other hand, the ordering $b, a, c$ does not respect the orbit structure and $\{1, b, a, c, b a, b c, a c, b a c\}$ will be a proper subset of $G$ (since $b a=a c$ and $b a c=a$ ).

Example 3.3. For a more complex example, consider the decorated graph in Figure 3 (the missing edges correspond to 2-periodic trajectories, hence commuting generators). One can show that this graph is admissible, hence defines a cube group $(G, S)$ of order $2^{8}=256$. The $G$-orbits are $T_{1}=\{a, b, e, f\}$ and $T_{2}=\{c, d, g, h\}$. The $G_{T_{1}}$-orbits are 

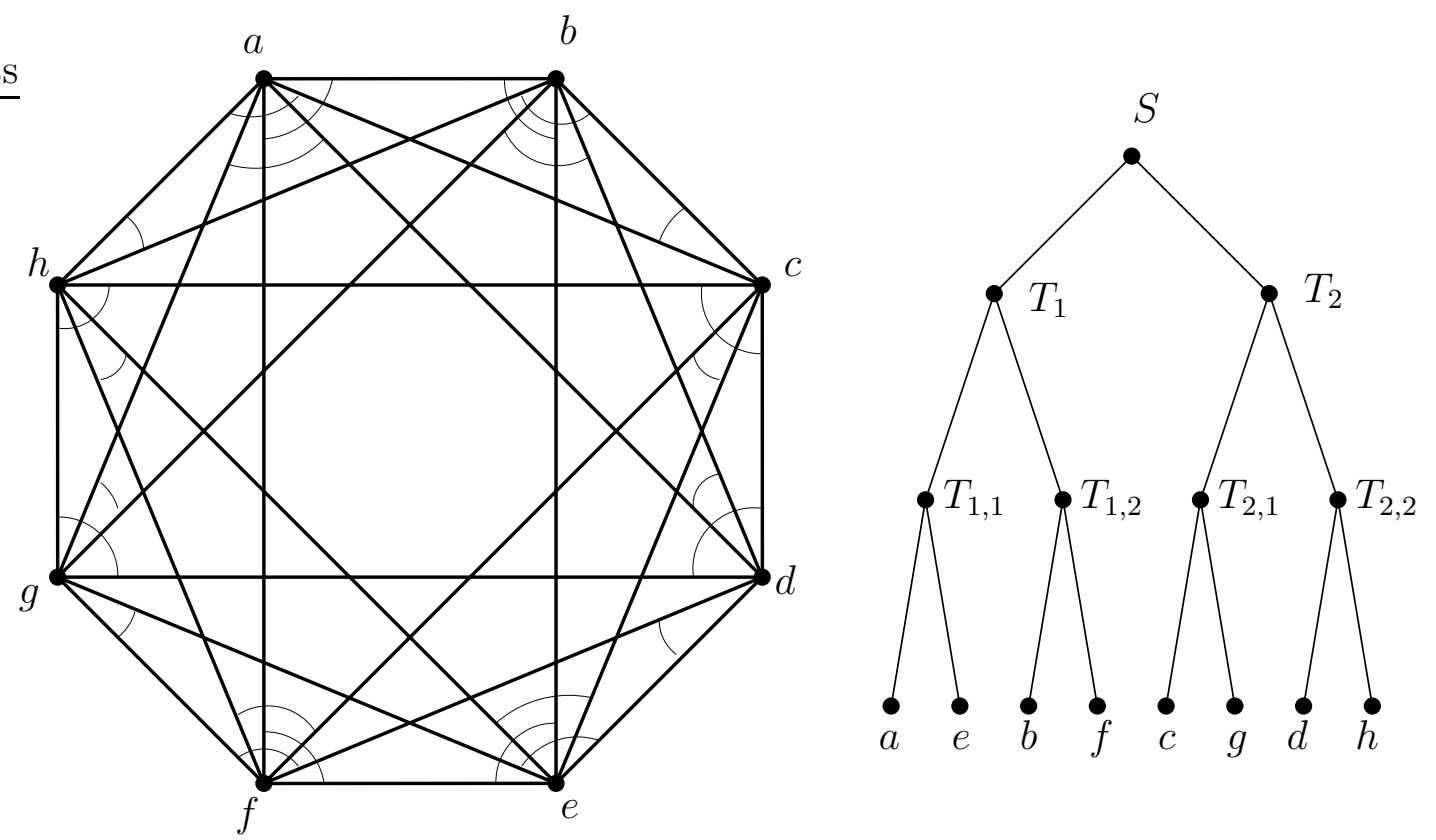

FiguRE 3.

$T_{1,1}=\{a, e\}, T_{1,2}=\{b, f\}$, and the $G_{T_{2}}$-orbits are $T_{2,1}=\{c, g\}, T_{2,2}=\{d, h\}$. Finally, all of these orbits break up into singleton orbits, so we can take as our decomposition

$$
G=G_{T_{1}} G_{T_{2}}=G_{T_{1,1}} G_{T_{1,2}} G_{T_{2,1}} G_{T_{2,2}}=\langle a\rangle\langle e\rangle\langle b\rangle\langle f\rangle\langle c\rangle\langle g\rangle\langle d\rangle\langle h\rangle .
$$

In fact any planar representation of the orbit tree shown in Figure 3 will give rise to a different product decomposition by reading off the final nodes from left to right.

\section{The geometric representation}

In this section we describe the geometric representation of a cube group $(G, S)$ arising from the left action of $G$ on $\operatorname{Cay}(G, S)$. We use the fact that the $n$-cube is rigid in the sense that any (graph) automorphism of its 1-skeleton is the restriction of an isometry of the entire cube.

Let $(G, S)$ be a cube group, and let $\mathbb{R}^{S}$ denote the finite dimensional real Euclidean space with standard basis $\left\{e_{s} \mid s \in S\right\}$. There is a natural identification of $\operatorname{Cay}(G, S)$ with the standard cube $[-1,1]^{S} \subseteq \mathbb{R}^{S}$ given as follows. Since Cay $(G, S)$ is isomorphic to the $n$-cube with $n=|S|$, we can index the vertices using subsets of $S$. More precisely, we let $g_{\emptyset}$ be the identity vertex. For any subset $T \subseteq S$, there is a unique minimal subcube containing the vertex $g_{\emptyset}$ and the vertices $s$ for all $s \in T$. We let $g_{T}$ denote the vertex opposite $g_{\emptyset}$ in this subcube. The embedding of $\operatorname{Cay}(G, S)$ into $\mathbb{R}^{S}$ is then given by the mapping

$$
g_{T} \mapsto v_{T}:=\sum_{s \notin T} e_{s}-\sum_{s \in T} e_{s}
$$

for all $T \subseteq S$. It follows from the way we indexed the elements of $G$ that the edges in the Cayley graph will map precisely to the edges in the 1 -skeleton of the cube $[-1,1]^{S}$. 
For the remainder of the paper, we shall identify $\operatorname{Cay}(G, S)$ with the 1-skeleton of the cube $[-1,1]^{S}$ via this embedding.

Let $(G, S)$ be a cube group. Any element $g \in G$ determines an automorphism of the Cayley graph $\operatorname{Cay}(G, S)$, hence an isometry of the cube $[-1,1]^{S}$. Any such isometry is the restriction of a unique orthogonal linear transformation $\rho_{g}: \mathbb{R}^{S} \rightarrow \mathbb{R}^{S}$. The resulting homomorphism $\rho: G \rightarrow G L\left(\mathbb{R}^{S}\right)$ taking $g$ to $\rho_{g}$ will be called the geometric representation of the pair $(G, S)$. Since $G$ acts simply-transitively on itself and $G$ is identified with the vertices of the cube $[-1,1]^{S}$, the geometric representation is obviously faithful.

The geometric representation can be described explicitly in terms of the permutation representation $j: G \rightarrow \operatorname{Aut}(S)$. Given a product representation $g=s_{k} s_{k-1} \cdots s_{1}$ for $g$ in terms of generators $s_{1}, s_{2}, \ldots s_{k} \in S$ and given any element $t \in S$, we let $n(g, t)$ denote the cardinality of the set

$$
\left\{i \in[1, k-1] \mid s_{i+1}=j_{s_{i}} j_{s_{i-1}} \cdots j_{s_{1}}(t)\right\} .
$$

We then obtain the following formula for $\rho$.

Proposition 4.1. Let $(G, S)$ be a cube group and let $\Gamma=\left\{j_{s} \mid s \in S\right\}$ be the corresponding decorated graph. For any $g \in G$, we let $j_{g} \in \operatorname{Aut}(S)$ denote the image of $g$ under the homomorphism $j$. The linear transformation $\rho_{g}: \mathbb{R}^{S} \rightarrow \mathbb{R}^{S}$ is then given by

$$
\rho_{g}\left(e_{t}\right)=(-1)^{n(g, t)} e_{j_{g}(t)} \text { for all } t \in S .
$$

(In particular, $n(g, s)$ is independent of the product representation of $g$ in terms of generators.)

Proof. First we prove that for any $s \in S$, we have

$$
\rho_{s}\left(e_{t}\right)= \begin{cases}-e_{t} & \text { if } t=s \\ e_{j_{s}(t)} & \text { if } t \neq s .\end{cases}
$$

For each $t \in S$, let $F_{t}^{+}$(respectively $F_{t}^{-}$) denote the facet (codimension-1 face) of the cube $[-1,1]^{S}$ with barycenter $e_{t}$ (resp., $-e_{t}$ ). The map $\rho_{s}$ must take the $n$ facets incident to the vertex 1 bijectively to the $n$ facets incident to the vertex $s$, permuting those facets incident to both 1 and $s$. The facets incident to 1 are precisely the "positive" ones $\left\{F_{t}^{+} \mid t \in S\right\}$, and the facets incident to $s$ are $\left\{F_{t}^{+} \mid t \neq s\right\} \cup\left\{F_{s}^{-}\right\}$. So $\rho_{s}$ must $\operatorname{map} F_{s}^{+}$to $F_{s}^{-}$, and hence for $t=s$ we have $\rho_{s}\left(e_{t}\right)=-e_{t}=-e_{j_{s}(t)}$.

Now suppose $t \neq s$, then the facet $F_{t}^{+}$is the unique facet containing the $(n-1)$ edges incident to the vertex 1 that are labeled by the set $\{u \mid u \neq t\}$. Equivalently, $F_{t}^{+}$is the unique facet containing the $(n-1)$ edges incident to the vertex $s$ that are labeled by the set $\left\{j_{s}(u) \mid u \neq t\right\}$ (see Figure 4). Since $\rho_{s}$ restricts to the left action of $s$ on the Cayley graph and this action preserves edge labels, it follows that $\rho_{s}\left(F_{s}^{+}\right)$is the unique facet spanned by the edges incident to the vertex $s$ that are labeled by the set $\{u \mid u \neq t\}$. This is the same as the facet spanned by the edges incident to the vertex 1 that are labeled by the set $\left\{j_{s}(u) \mid u \neq t\right\}$, or equivalently, the set $\left\{u \mid u \neq j_{s}(t)\right\}$. But this is precisely the facet $F_{j_{s}(t)}^{+}$. Restricting $\rho_{s}$ to the barycenters of these facets, we then have $\rho_{s}\left(e_{t}\right)=e_{j_{s}(t)}$. 


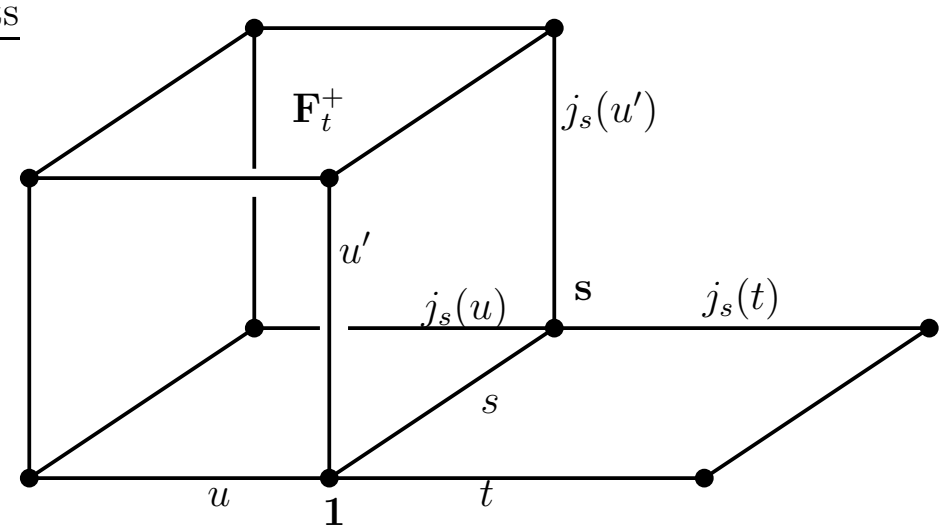

Figure 4.

Finally, suppose $g=s_{k} \cdots s_{2} s_{1}$. Then

$$
\begin{aligned}
\rho_{g}\left(e_{t}\right)= & \rho_{s_{k}} \cdots \rho_{s_{2}} \rho_{s_{1}}\left(e_{t}\right) \\
= & \rho_{s_{k}} \cdots \rho_{s_{2}}\left((-1)^{n\left(s_{1}, t\right)} e_{j_{s_{1}}(t)}\right) \\
= & \rho_{s_{k}} \cdots \rho_{s_{3}}\left((-1)^{n\left(s_{2} s_{1}, t\right)} e_{\left.j_{s_{2}} j_{s_{1}}(t)\right)}\right. \\
& \quad \vdots \\
= & (-1)^{n\left(s_{k} \cdots s_{1}, t\right)} e_{j_{s_{k}} \cdots j_{s_{2}} j_{s_{1}}(t)} \\
= & (-1)^{n(g, t)} e_{j_{g}(t)}
\end{aligned}
$$

as desired.

It follows immediately from this formula for the geometric representation $\rho$ that if $T \subseteq S$ is a $G$-invariant subset of $S$, then $\mathbb{R}^{T} \subseteq \mathbb{R}^{S}$ is an invariant subspace for $\rho$. By Theorem $3.1, S$ always admits a proper nontrivial $G$-invariant subset, hence we have our second theorem from the introduction.

Corollary 4.1. If $(G, S)$ is a cube group, then the geometric representation $\rho$ is reducible.

\section{References}

[1] R. Scott. Right-angled mock reflection and mock Artin groups. Trans. Amer. Math. Soc. 360 (2008), no. 8, 4189-4210.

[2] H. Wielandt. Ein Beweis für die Existenz der Sylowgruppen. Arch. Math. (Basel) 10 (1959), 401-402.

Department of Mathematics and Computer Science, Santa Clara University, Santa Clara, CA 95053

E-mail address: rscott@scu.edu 